\title{
Plane waves in a rotating generalized thermo-elastic solid with voids
}

\author{
Jaswant Singh ${ }^{1}$, S.K. Tomar ${ }^{2 *}$ \\ ${ }^{1}$ Department of Mathematics, Government Post Graduate College, Nalagarh-174 101, (HP) INDIA \\ ${ }^{2 *}$ Department of Mathematics, Panjab University, Chandigarh-160 014, INDIA \\ *Corresponding Author: e-mail:sktomar66@gmail.com, Tel +91-172-2534523, Fax. +91-172-2541132
}

\begin{abstract}
Propagation of plane waves in a rotating thermo-elastic solid with voids has been studied. The theory for thermo-elastic materials with voids developed by Iesan in the context of thermo- elastic theory of Lord and Shulman has been employed for mathematical treatment. It has been found that there exist one transverse wave and three sets of coupled longitudinal waves. Each set of coupled longitudinal waves is found to be influenced by the rotation, presence of voids and thermal property of the solid, whereas the transverse wave is found to be influenced only by the rotation of the body and remains un-affected by the presence of voids and thermal parameters. Numerical treatment is given to seek these effects on phase speeds of the existed waves.
\end{abstract}

Keywords: Thermo-elastic, voids, rotation, plane, waves.

\section{Introduction}

Nunziato and Cowin (1979) and Cowin and Nunziato (1983) developed nonlinear and linear theory of elastic material with voids by using the concept of distributed body introduced by Goodman and Cowin (1972). In the linear theory of elastic material with voids, the change in void volume fraction and strain are taken as independent kinematic variables. Puri and Cowin (1985) showed that there exist two dilational waves in porous elastic material with voids, one of them is predominantly the dilational wave of classical linear elasticity and the other is predominantly a wave carrying a change in the void volume fraction. Both the waves are found to attenuate in their direction of propagation. They found that at large frequency, the predominantly elastic wave propagates with the classical elastic dilational wave speed, but at low frequency it propagates at a speed less than the classical wave speed.

Iesan (1986) presented a linear theory for thermo-elastic material with voids. He derived the basic equations and proved the uniqueness of solution, reciprocity relation and variational characterization of solution in the dynamical theory. Later, Cicco and Diaco (2002) presented a theory of thermo-elastic material with voids without energy dissipation. A number of problems related to wave propagation in the elastic material with voids have been attempted by various authors in the past and they have appeared in the open literature. Some of them are Chandersekharaiah (1987), Wright (1998), Ciarletta and Sumbatyan (2003), Dey et al. (2003), Singh and Tomar (2007a, b). Keeping in view that the propagation of waves in rotating media are important in many realistic problems, e.g., rotation of heavenly bodies, the present problem of plane waves in a generalized thermo-elastic solid with voids rotating with uniform angular velocity has been investigated. It has been found that there exist one transverse wave and three sets of coupled longitudinal waves in a rotating homogeneous generalized thermo-elastic solid with void. Each set of coupled longitudinal waves is found to be influenced by the rotation, voids and thermal property of the solid, whereas the transverse wave is found to be influenced only by the rotation of the body and it remains un-influenced by the presence of voids and thermal parameters. 


\section{Propagation of plane waves}

The field equations in term of displacement, volume fraction and temperature for generalized thermo-elastic solid with voids and rotating with uniform angular velocity $\boldsymbol{\Omega}$, in the absence of body forces, heat and extrinsic equilibrated body forces are given by (see Iesan, 1986; Lord and Shulman, 1967)

$$
\begin{aligned}
& \mu \nabla^{2} \mathbf{u}+(\lambda+\mu) \nabla(\nabla \cdot \mathbf{u})-\beta \nabla \Theta+b \nabla \phi=\rho[\ddot{\mathbf{u}}+\mathbf{\Omega} \times(\boldsymbol{\Omega} \times \mathbf{u})+2 \Omega \times \dot{\mathbf{u}}], \\
& \rho C_{e}\left(\dot{\Theta}+\tau_{0} \ddot{\Theta}\right)+\beta T_{0}\left(\nabla \cdot \dot{\mathbf{u}}+\tau_{0} \nabla \cdot \ddot{\mathbf{u}}\right)+m T_{0}\left(\dot{\phi}+\tau_{0} \ddot{\phi}\right)=K \nabla^{2} \Theta, \\
& \alpha \nabla^{2} \phi-\xi \phi-b \nabla \cdot \mathbf{u}+m \Theta=\rho \chi \ddot{\phi},
\end{aligned}
$$

where $\lambda$ and $\mu$ are Lame's parameters, $\rho$ is the density of the medium, $\Theta=T-T_{0}, T_{0}$ being the temperature of the medium in its natural state and assumed to be such that $\left|\Theta / T_{0}\right| \leq 1, T$ is the temperature, $K$ is the coefficient of thermal conductivity, $\tau_{0}$ is the thermal relaxation time, $C_{e}$ is the specific heat at constant strain. The quantities $\alpha, b$ and $\xi$ are the void parameters; $m$ is the thermo-void coefficient, $\chi$ is the equilibrated inertia, $\beta=(3 \lambda+2 \mu) \alpha_{t}, \alpha_{t}$ being the coefficient of linear thermal expansion, $\mathbf{u}(\mathbf{x}, t)$ is the displacement vector and $\phi$ is the change in void volume fraction. The term " $\boldsymbol{\Omega} \times(\boldsymbol{\Omega} \times \mathbf{u})$ " represents the centripetal acceleration, while the term " $2 \boldsymbol{\Omega} \times \dot{\mathbf{u}}$ " represents Coriolis acceleration (see Chandrasekharaiah, 1987). It can be seen that by neglecting these terms, one can recover the equations of motion for a non-rotating thermo-elastic body with voids given by Iesan (1986) in the context of Lord and Shulman (1967).

For plane waves propagating in the positive direction of a unit vector $\mathbf{n}$, we may take

$$
\{\mathbf{u}, \Theta, \phi\}=\{\mathbf{A}, B, C\} \exp [i k(\mathbf{n} \cdot \mathbf{r}-v t)]
$$

where $\mathbf{A}$ is vector constant, $B$ and $C$ are scalar constants representing the amplitudes, $\mathbf{r}$ is the position vector, $v$ is the phase speed and $k$ is the wavenumber having the definition that $\omega=k v, \omega$ being the circular frequency. On inserting (4) into equations (1) - (3), we obtain

$$
\begin{aligned}
& \left(\Omega^{2}+\omega^{2}-c_{2}^{2} k^{2}\right) \mathbf{A}-\left\{\left(c_{1}^{2}-c_{2}^{2}\right) k^{2}(\mathbf{n} \cdot \mathbf{A})+i \beta^{*} k B-i b^{*} k C\right\} \mathbf{n}-\{(\mathbf{\Omega} \cdot \mathbf{A}) \mathbf{\Omega}+2 i \omega(\mathbf{\Omega} \times \mathbf{A})\} \\
& \quad=\mathbf{0}, \\
& i k \delta_{0} v^{2}(\mathbf{n} \cdot \mathbf{A})+\left(v^{2}-\frac{\eta_{o}}{\tau_{1}}\right) B+\sigma_{0} v^{2} C=0,
\end{aligned}
$$

$\frac{i v^{*}}{k}(\mathbf{n} \cdot \mathbf{A})-\frac{m^{*}}{k^{2}} B-\left(v^{2}-c_{3}^{2}-\frac{\xi^{*}}{k^{2}}\right) C=0$

where

$$
\begin{aligned}
& c_{1}^{2}=\frac{\lambda+2 \mu}{\rho}, \quad c_{2}^{2}=\frac{\mu}{\rho}, \quad c_{3}^{2}=\frac{\alpha}{\rho \chi}, \quad \xi^{*}=\frac{\xi}{\rho \chi}, \quad m^{*}=\frac{m}{\rho \chi}, \quad \beta^{*}=\frac{\beta}{\rho}, \\
& b^{*}=\frac{b}{\rho}, \quad \delta_{0}=\frac{\beta T_{0}}{\rho C_{e}}, \quad \sigma_{0}=\frac{m T_{0}}{\rho C_{e}}, \quad \eta_{0}=\frac{K}{\rho C_{e}}, \quad \tau_{1}=\tau_{0}+\frac{i}{\omega}, \quad v^{*}=\frac{b}{\rho \chi} .
\end{aligned}
$$

Solving equations (6) and (7) for the constants $B$ and $C$ and substituting their values in equation (5), we obtain the following vector equation 


$$
\begin{gathered}
\left(\Omega^{2}+\omega^{2}-c_{2}^{2} k^{2}\right) \mathbf{A}-\left\{\left(c_{1}^{2}-c_{2}^{2}\right) k^{2}+i \beta^{*} k J_{i}-i b^{*} k H_{i}\right\}(\mathbf{n} \cdot \mathbf{A}) \mathbf{n}-\{(\boldsymbol{\Omega} \cdot \mathbf{A}) \boldsymbol{\Omega}+2 i \omega(\boldsymbol{\Omega} \times \mathbf{A})\} \\
=\mathbf{0},
\end{gathered}
$$

where

$$
\begin{aligned}
& J_{i}=\frac{i}{k}\left[\left(\delta_{0} \xi^{*} \sigma_{0}-v^{*}-\delta_{0} \omega^{2}\right) v^{2}+\delta_{0} \omega^{2} c_{3}^{2}\right] \\
& \times\left.\times\left\{1-\frac{1}{\omega^{2}}\left(\xi^{*}+\sigma_{0} m^{*}\right)\right\} v^{4}-\left\{c_{3}^{2}+\frac{\eta_{0}}{\tau_{1}}\left(1-\frac{\xi^{*}}{\omega^{2}}\right)\right\} v^{2}+\frac{\eta_{0}}{\tau_{1}} c_{3}^{2}\right]^{-1}, \\
& H_{i}=\frac{i}{k}\left[\left(m^{*} \delta_{0}+v^{*}\right) v^{*}+\frac{\eta_{0}}{\tau_{1}} v^{*}\right] \\
& \times\left[\left\{1-\frac{1}{\omega^{2}}\left(\xi^{*}+\sigma_{0} m^{*}\right)\right\} v^{4}-\left\{c_{3}^{2}+\frac{\eta_{0}}{\tau_{1}}\left(1-\frac{\xi^{*}}{\omega^{2}}\right)\right\} v^{2}+\frac{\eta_{0}}{\tau_{1}} c_{3}^{2}\right]^{-1} .
\end{aligned}
$$

Taking scalar product of equation (8) with vector $\mathbf{A}$, we obtain

$\left(\Omega^{2}+\omega^{2}-c_{2}^{2} k^{2}\right) \mathrm{A}^{2}-\left\{\left(c_{1}^{2}-c_{2}^{2}\right) k^{2}+i \beta^{*} k J_{i}-i b^{*} k H_{i}\right\}(\mathbf{n} \cdot \mathbf{A})^{2}-\left\{(\boldsymbol{\Omega} \cdot \mathbf{A})^{2}+\mathbf{2 i \omega}(\boldsymbol{\Omega} \times \mathbf{A}) \cdot \mathbf{A}\right\}=$ 0 ,

where $A=|\mathbf{A}|$.

For a plane transverse wave, we have $\mathbf{n} \cdot \mathbf{A}=A$ and the equation (9) yields the speed of transverse wave given by

$v_{T}=\frac{c_{2}}{R}, \quad R=\sqrt{1+\frac{\Omega^{2}}{\hat{\mathrm{e}}^{2}} \sin ^{2} \theta,}$

where $\theta$ is the angle between the axis of rotation of the body and the direction of the displacement vector. If $\theta=0$, then $v_{T}=c_{2}$. This means that when the axis of rotation of the body is parallel to the direction of the displacement vector, then the phase speed of transverse wave remains independent of the rotation of the body. We also note that for large angular velocity of the body, i.e., when $\Omega \rightarrow \infty$, the phase speed $v_{T} \rightarrow 0$. This means that transverse waves are not allowed to propagate for bodies rotating at very high speed.

For a plane longitudinal wave, we have $\mathbf{n} \cdot \mathbf{A}=\mathrm{A}$ and the equations (9) yields the following six degree equation in $v$ :

$a_{0} v^{6}+a_{1} v^{4}+a_{2} v^{2}+a_{3}=0$,

where

$$
\begin{aligned}
& a_{0}= R^{2}\left[1-\frac{1}{\omega^{2}}\left(\xi^{*}+\sigma_{0} m^{*}\right)\right] \\
& a_{1}=-\left\{c_{1}^{2}+c_{3}^{2} R^{2}+\frac{\eta_{0} R^{2}}{\tau_{1}}\left(1-\frac{\xi^{*}}{\omega^{2}}\right)+\beta^{*} \delta_{0}-\frac{1}{\omega^{2}}\left(c_{1}^{2} \xi^{*}-\delta_{0} b^{*} m^{*}+\delta_{0} \beta^{*} \xi^{*}+\sigma_{0} c_{1}^{2} m^{*}-\beta^{*} \sigma_{0} v^{*}\right.\right. \\
&\left.\left.\quad-b^{*} v^{*}\right)\right\} \\
& a_{2}=\left(c_{1}^{2}+\delta_{0} \beta^{*}\right) c_{3}^{2}+\frac{\eta_{0}}{\tau_{1}}\left[c_{1}^{2}+c_{3}^{2} R^{2}-\frac{1}{\omega^{2}}\left(c_{1}^{2} \xi^{*}-v^{*} b^{*}\right)\right] \\
& a_{3}=-\frac{\eta_{0}}{\tau_{1}} c_{1}^{2} c_{3}^{2} .
\end{aligned}
$$


Using Cardan's method in equation (11), we obtain

$Z^{3}+3 H Z+G=0$,

where

$Z=a_{0} v^{2}+\frac{a_{1}}{3}, \quad H=\frac{a_{0} a_{2}}{3}-\frac{a_{1}^{2}}{9}$ and $\quad G=\frac{2 a_{1}^{3}}{27}-\frac{a_{0} a_{1} a_{2}}{3}+a_{0}^{2} a_{3}$.

The three roots of equation (12) are given by

$$
Z_{1}=S, \quad Z_{2}=\frac{1}{2}(-S+i \sqrt{3} T), \quad Z_{3}=\frac{1}{2}(-S-i \sqrt{3} T) .
$$

where

$$
S=U+V, \quad T=U-V, \quad U^{3}=\frac{1}{2}\left[-G+\sqrt{G^{2}+4 H^{3}}\right] \quad \text { and } \quad V=-\frac{H}{U} .
$$

Hence, the three roots of equation (11) are given by

$$
v_{1}^{2}=\frac{1}{a_{0}}\left(S-\frac{a_{1}}{3}\right), v_{2}^{2}=\frac{1}{a_{0}}\left(-\frac{1}{2} S+\frac{\sqrt{3}}{2} i T-\frac{a_{1}}{3}\right), \quad v_{3}^{2}=\frac{1}{a_{0}}\left(-\frac{1}{2} S-\frac{\sqrt{3}}{2} i T-\frac{a_{1}}{3}\right) .
$$

\section{Numerical observations}

To study the effect of rotation of the body on the phase speeds of the various existed waves, we have computed these phase speeds by using following values of the relevant parameters (see Dhaliwal and Singh, 1980, pp:726) for cobalt, magnesium and zinc crystal like materials. These materials are modelled as isotropic generalized thermo-elastic materials with voids.

\begin{tabular}{|cllll|}
\hline Symbol & Cobalt & Magnesium & \multicolumn{1}{c|}{ Zinc } & Unit \\
\hline$\rho$ & $8.836 \times 10^{3}$ & $1.74 \times 10^{3}$ & $7.14 \times 10^{3}$ & $\mathrm{kgm}^{-3}$ \\
$\lambda$ & $1.027 \times 10^{11}$ & $2.17 \times 10^{10}$ & $0.508 \times 10^{11}$ & $\mathrm{Nm}^{-2}$ \\
$\mu$ & $1.510 \times 10^{11}$ & $3.278 \times 10^{10}$ & $0.770 \times 10^{11}$ & $\mathrm{Nm}^{-2}$ \\
$\beta$ & $7.07 \times 10^{6}$ & $2.68 \times 10^{6}$ & $5.75 \times 10^{6}$ & $\mathrm{Nm}^{-2} \mathrm{deg}^{-1}$ \\
$C_{e}$ & $4.27 \times 10^{2}$ & $1.04 \times 10^{3}$ & $3.9 \times 10^{2}$ & $\mathrm{~J} \mathrm{~kg}^{-1} \mathrm{deg}^{-1}$ \\
$K$ & $0.690 \times 10^{2}$ & $1.7 \times 10^{2}$ & $1.24 \times 10^{2}$ & $\mathrm{Wm}^{-1} \mathrm{deg}^{-1}$ \\
$\tau_{0}$ & $0.18 \times 10^{-12}$ & $0.02 \times 10^{-11}$ & $0.011 \times 10^{-11}$ & $\mathrm{sec}^{11}$ \\
$\omega$ & $1.88 \times 10^{12}$ & $3.58 \times 10^{11}$ & $5.01 \times 10^{11}$ & $\mathrm{sec}^{-1}$ \\
$T_{o}$ & $0.0298 \times 10^{4}$ & $0.0298 \times 10^{4}$ & $0.0296 \times 10^{4}$ & $\mathrm{~K}^{0}$ \\
\hline
\end{tabular}

The numerical values of the relevant parameters corresponding to voids in these materials are taken same. These are considered as

\begin{tabular}{|ccl|}
\hline Symbol & Value & \multicolumn{1}{c|}{ Unit } \\
\hline$\alpha$ & $3.688 \times 10^{-5}$ & $\mathrm{~N}$ \\
$m$ & $2 \times 10^{6}$ & $\mathrm{Nm}^{-2} \mathrm{deg}^{-1}$ \\
$b$ & $1.13849 \times 10^{10}$ & $\mathrm{Nm}^{-2}$ \\
$\xi$ & $1.473 \times 10^{10}$ & $\mathrm{Nm}^{-2}$ \\
$\chi$ & $1.753 \times 10^{-15}$ & $\mathrm{~m}^{2}$ \\
\hline
\end{tabular}

In the figures, the curves with black, red and blue inks represent the phase speed in cobalt, magnesium and zinc crystals, respectively. The solid curves correspond to the case of a non-rotating body, while the dotted curves correspond to the case of rotating body. 


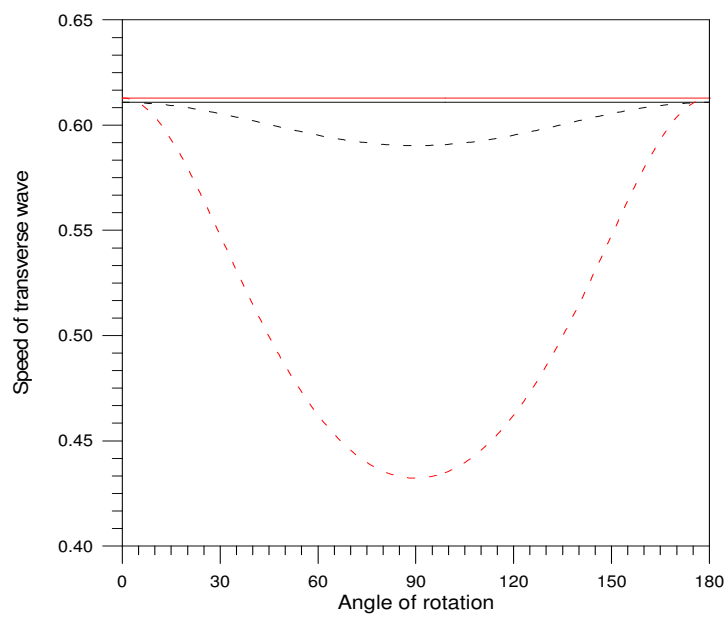

Figure 1: Angle of rotation versus phase speed of transverse wave in a single crystal of Cobalt and Magnesium.

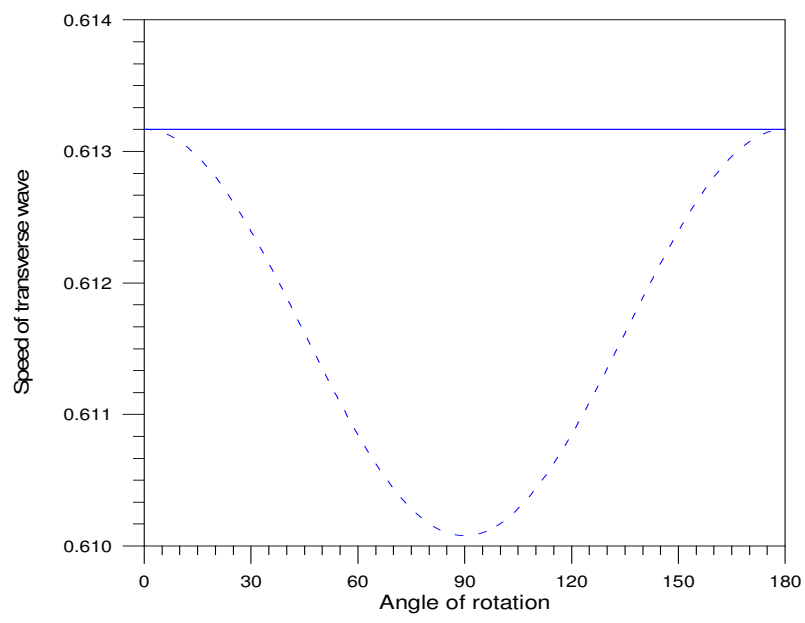

Figure 2: Angle of rotation versus phase speed of transverse wave in single crystal of Zinc.

Figures 1 and 2 depict the effect of rotation of the body on the phase speed of transverse wave. For all the three materials, it is found that with the increase of the angle of rotation between the displacement vector and the axis of rotation of the material body, the phase speed of the transverse wave decreases till the transverse wave propagates perpendicular to the axis of rotation of the body. Thereafter, its phase speed increases till the wave travels parallel to the axis of the rotation of the body. The effect of rotation of the body on the phase speed of transverse wave is different in different materials. It is noted that for the case of non rotating bodies, the phase speed of transverse wave is almost same in all the three materials, while it is significantly affected by the rotation of the material.

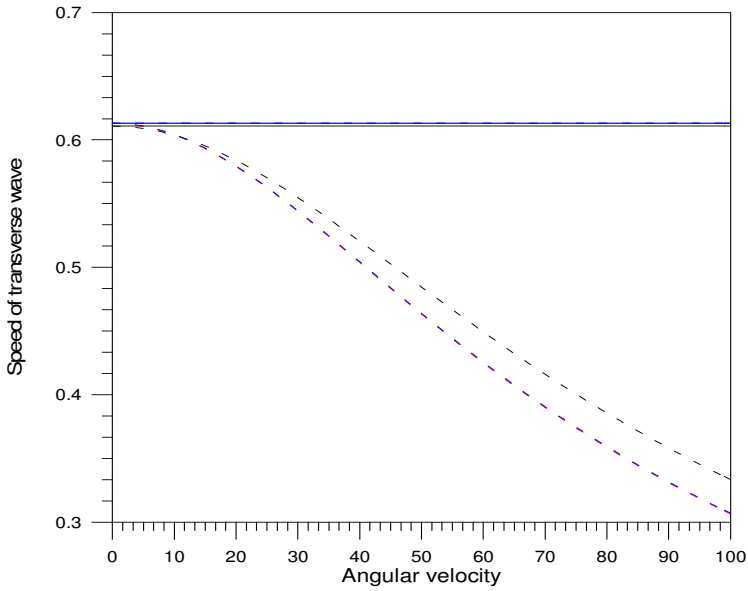

Figure 3: Angular velocity versus phase speed of transverse wave in a single crystal of Cobalt, Magnesium and Zinc, respectively(when $\theta=60^{\circ}$ ).

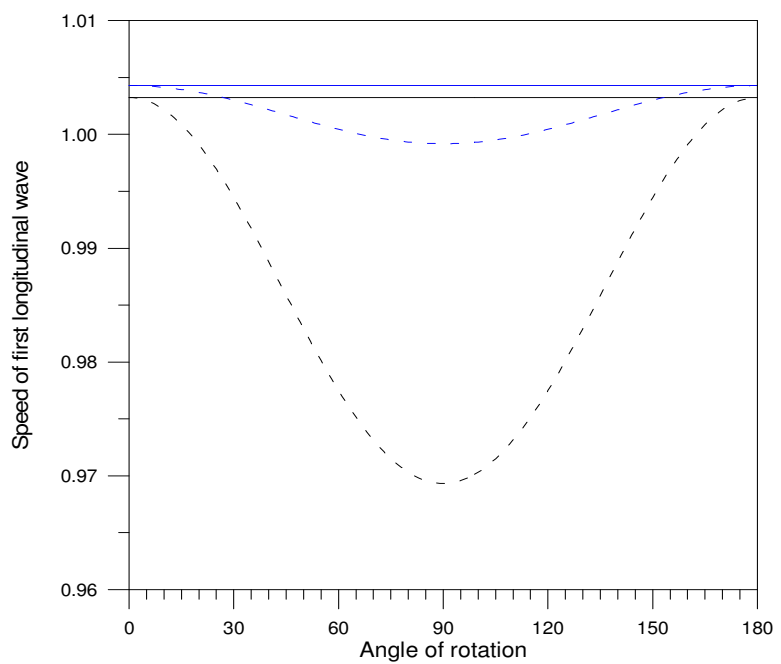

Figure 4: Angle of rotation versus phase speed of first longitudinal wave in a single crystal of Cobalt and Zinc.

Figure 3 depicts the effect of angular velocity of the body on the phase speed of transverse wave. It is found that with the increase of angular velocity of the body, the phase speed of the transverse wave decreases. Although, the phase speed of the transverse wave remains almost same in all the three materials considered for non-rotating case, but with the increase of the angular velocity of the body, the rate of decrease is same in magnesium and zinc while decreases with different rate in cobalt crystal. However, as the angular velocity take large and large values, the phase speed of transverse wave approaches to zero as was observed analytically.

Figure 4 depicts the effect of rotation of the body on the phase speed of first longitudinal wave in cobalt and zinc. It is clear from this figure that this wave travels faster in zinc material than the cobalt material. In both the materials, the effect of rotation on the phase speed of this wave is similar as in the case of transverse wave. 


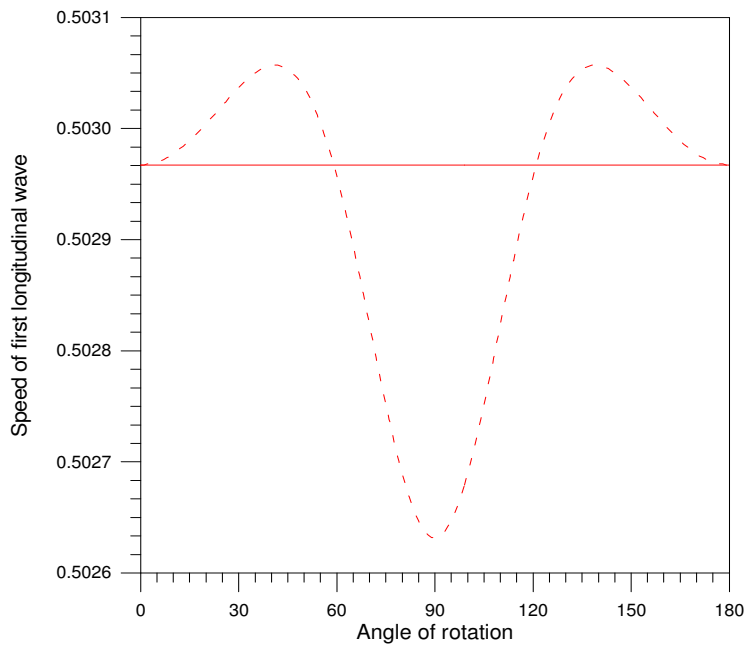

Figure 5: Angle of rotation versus phase speed of first longitudinal wave in a single crystal of Magnesium.

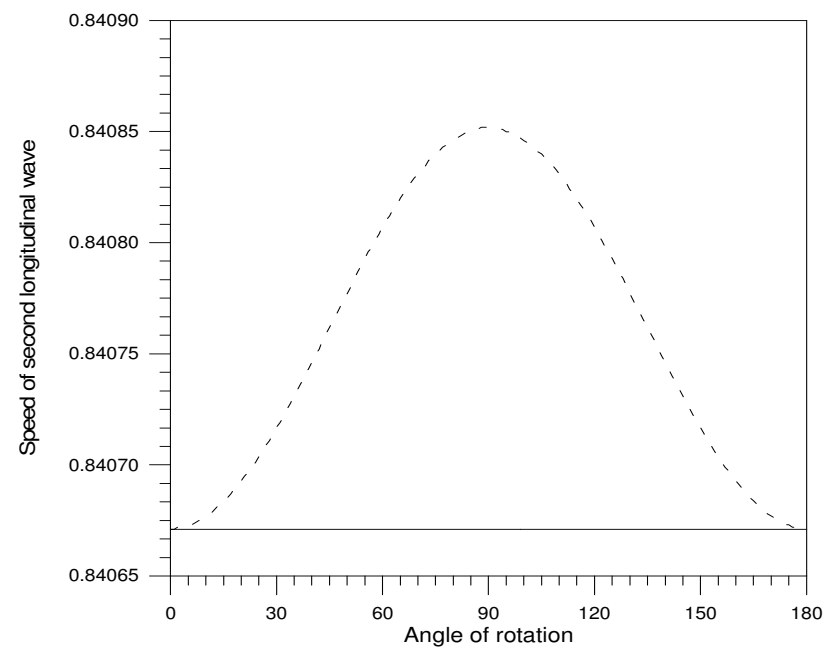

Figure 6: Angle of rotation versus phase speed of second longitudinal wave in a single crystal of Cobalt.

Figure 5 depicts the effect of rotation of the body on the phase speed of first longitudinal wave in magnesium crystal. This effect is little different than that of in the cobalt and zinc materials. It is found that initially in the range $0^{0}-45^{0}$, the phase speed of this wave increases with the increase of angle of rotation and then decreases rapidly in the range $45^{\circ}-90^{\circ}$. Thereafter, the trend is reverse but symmetric.

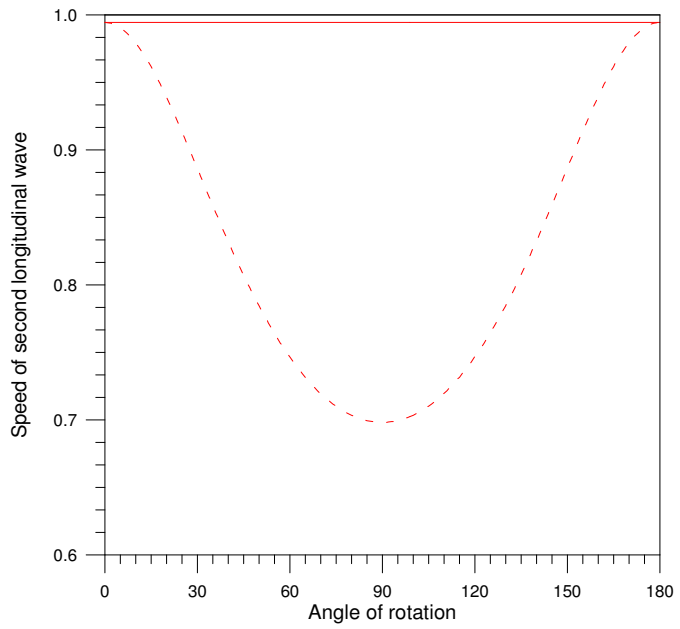

Figure 7: Angle of rotation versus phase speed of second longitudinal wave in a single crystal of Magnesium.

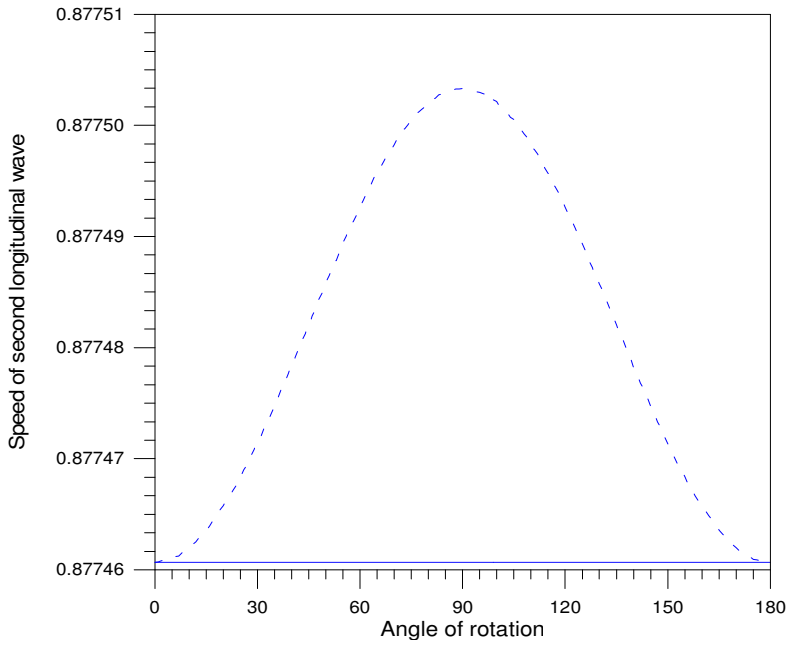

Figure 8: Angle of rotation versus phase speed of second longitudinal wave in a single crystal of Zinc.

Figures 6, 7 and 8 depict the effect of rotation of the body on the phase speed of second longitudinal wave in cobalt, magnesium and zinc crystal respectively. It is noted from these figures that in the cobalt and zinc crystal, the phase speed of second longitudinal wave increases with the increase of the angle of rotation, while it decreases in magnesium crystals. The effect of rotation on this wave is minimum at $0^{0}$ angle and is maximum at $90^{\circ}$ angle. 


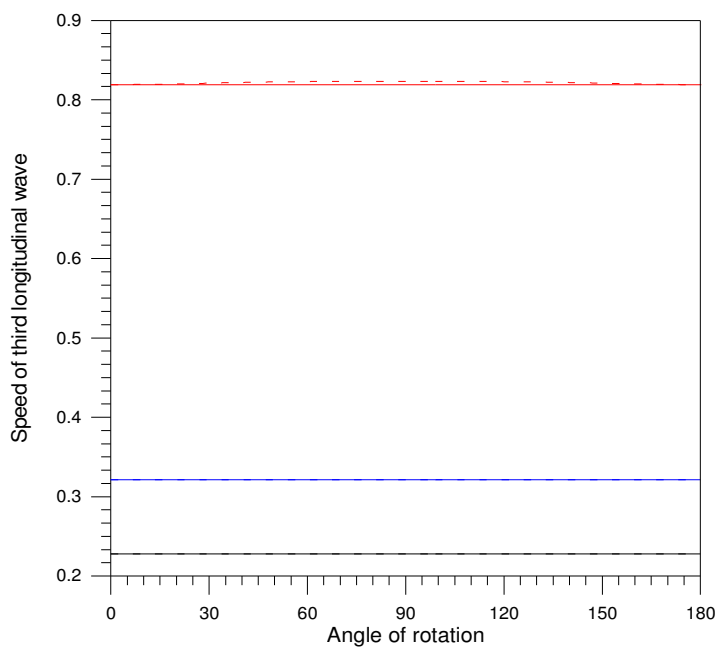

Figure 9: Angle of rotation versus phase speed of third longitudinal wave in a single crystal of Cobalt, Magnesium and Zinc respectively.

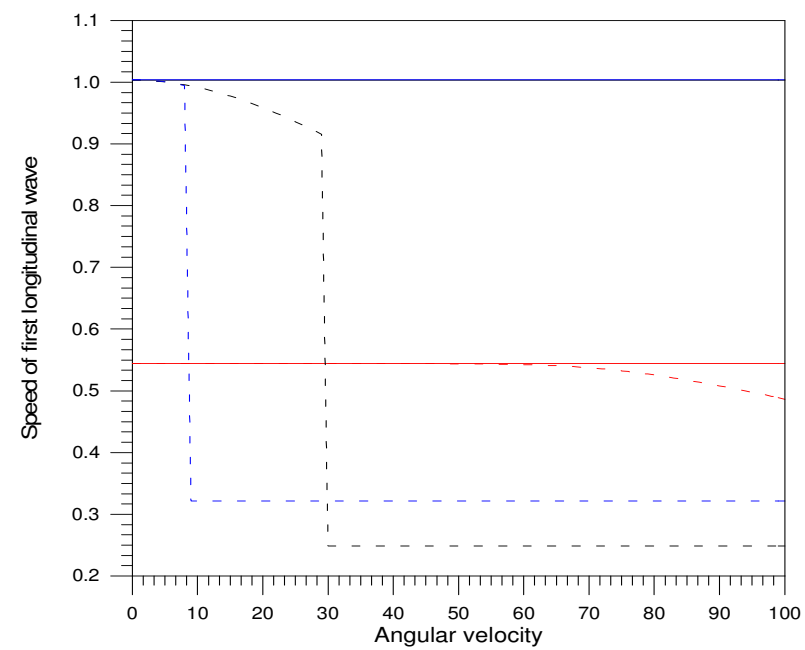

Figure 10: Angular velocity versus phase speed of first longitudinal wave in the single crystal of Cobalt, Magnesium and Zinc respectively (when $\theta=60^{\circ}$ ).

Figure 9 depicts the effect of angle of rotation on the phase speed of third longitudinal wave. It is noted from this figure that the rotation of the body increases the phase speed of this wave in magnesium crystal; however it has no effect in cobalt and zinc crystal. Also, it is found that the magnitude of phase speed of this wave in cobalt and zinc crystal is less than that of in magnesium crystal.

Figures 10,11 and 12 depict the effect of angular velocity of the body on the phase speed of first, second and third longitudinal waves in cobalt, magnesium and zinc crystal respectively. It is noted from Figure 10 that the phase of speed of first longitudinal wave, in all the three materials decreases with the increase of the angular velocity of the body. Figure 11 indicates that the phase speed of second longitudinal wave decreases in magnesium, while in cobalt and zinc crystal, it first increases and then decreases towards a minimum constant value with further increase of the angular velocity of the body. From Figure 12, we see that in cobalt and zinc crystals, the phase speed of third longitudinal wave first increases and then obtain constant value while it has no effect for magnesium crystal.

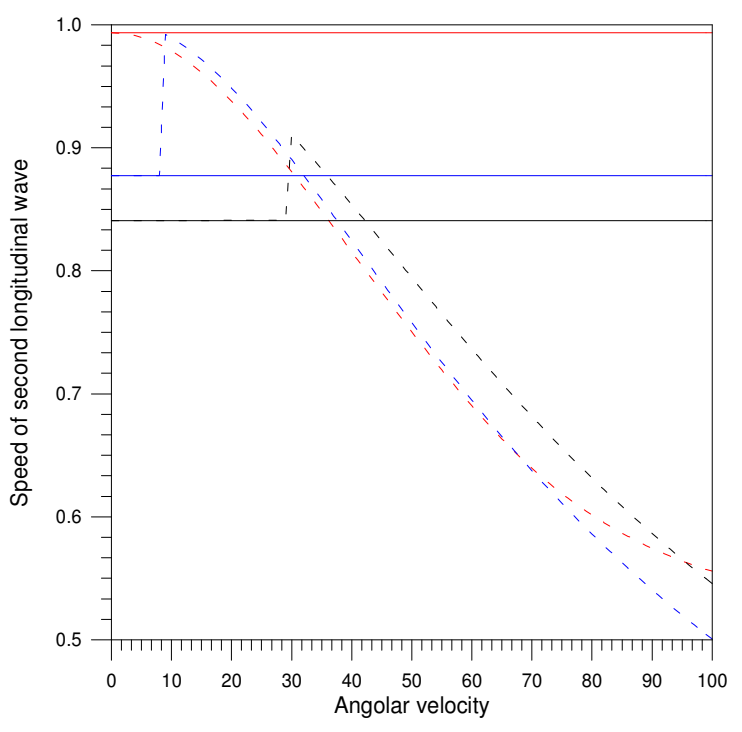

Figure 11: Angular velocity versus phase speed of second longitudinal wave in the single crystal of Cobalt, Magnesium and Zinc respectively. (when $\theta=60^{\circ}$ ).

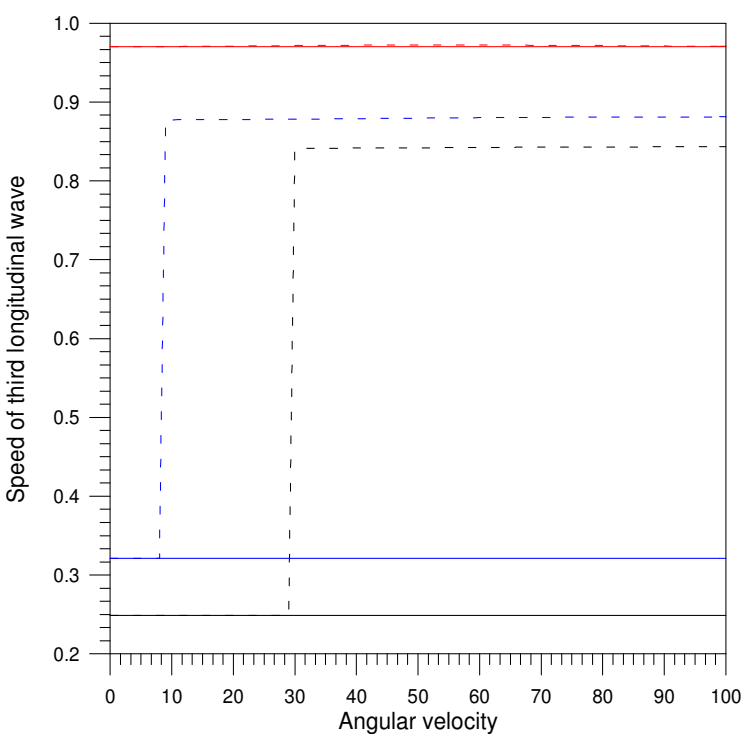

Figure 12: Angular velocity versus phase speed of third longitudinal wave in the single crystal of Cobalt, Magnesium and Zinc respectively (when $\theta=60^{\circ}$ ). 


\section{Conclusions}

In this article, we have studied the wave propagation in a generalized thermo-elastic solid with voids and rotating with uniform angular velocity. The results concluded from the above analysis can be summarized as:

1. It is found that there can exist four plane waves in a generalized thermo-elastic porous solid rotating with a uniform angular velocity. Out of the four waves; one is a transverse wave and the remaining three waves are three sets of coupled longitudinal waves.

2. The transverse wave is affected by the rotation of the body but remains independent by the presence of the voids and thermal property of the medium, as in the case of non-rotating body.

3. Each set of the longitudinal waves, is found to be influenced by the presence of void volume fraction and the thermal properties of the body. These sets are also found to be affected by the rotation of the body.

4. The rotation of the body has no effect on all the existed waves when they travel parallel to the axis of rotation of the body, while the influence is found to be maximum when they travel normal to the axis of rotation of the body.

\section{References}

Cowin S. C., Nunziato J. W., 1983. Linear theory of elastic material with voids. Journal of Elasticity, Vol. 13, pp. 125 - 147.

Chandrasekharaiah D.S., 1987. Plane waves in a rotating elastic solid with voids. International Journal of Engineering Science, Vol. 25, pp. 591 - 596.

Ciarletta M., Sumbatyan M. A., 2003. Reflection of plane waves by the free boundary of a porous elastic half-space. Journal of Sound and Vibration, Vol. 259, No. 2, pp. 253 - 264.

Cicco S.D., Diaco M., 2002. A theory of thermo-elastic material with voids without energy dissipation. Journal of Thermal Stresses, Vol. 25, pp. 493 - 503.

Dey S., Gupta S., Gupta A. K., Kar S. K., DE P. K., 2003. Propagation of torsional surface waves in an elastic layer with void pores over an elastic half-space with void pores. Tamkang Journal of Science and Engineering, Vol. 6, No. 4, pp. 241 - 249.

Dhaliwal R. S., Singh A., 1980. Dynamic Coupled Thermoelasticity. Hindustan Publishing Corporation, New Delhi, India.

Goodman M. A., Cowin S. C., 1972. A continuum theory for granular material. Archive for Rational Mechanics and Analysis, Vol. 44, pp. $248-265$.

Iesan D., 1986. A theory of thermo-elastic material with voids. Acta Mechanica, Vol. 60, pp. 67 - 89.

Lord H.W., Shulman Y., 1967. A generalized dynamical theory of thermo-elasticity. Journal of Mechanics and Physics of Solids, Vol. 15, pp. $299-309$.

Puri P., Cowin S. C., 1985. Plane waves in linear elastic material with voids. Journal of Elasticity, Vol. 15, pp. 167 - 183.

Singh J., Tomar S. K., 2007a. Plane waves in thermo- elastic material with voids. Mechanics of Materials, Vol. 39, pp. 932 - 940.

Singh J., Tomar S. K., 2007b. Plane waves in a rotating micro polar porous elastic solid. Journal of Applied Physics, Vol. 102, No.1.

Wright T. W., 1998. Elastic wave propagation through a material with voids. Journal of Mechanics and Physics of Solids, Vol. 46, No. 10, pp. 2033 - 2047.

\section{Biographical notes}

Dr. J. Singh received his M. Sc. degree in 1995 from Himachal Pradesh University, Shimla, (Himachal Pradesh) and Ph.D. degree in 2010from Panjab University, Chandigarh. Presently, he is an Assistant Professor in the Department of Mathematics, Post Graduate College, Nalagarh (HP), India.

Dr. S.K. Tomar is a Professor in the Department of Mathematics, Panjab University, Chandigarh (UT), India. He has more than twenty three years of experience in teaching and research. His current area of research includes Waves and Vibrations in continuous media, Theoretical Seismology, Numerical computations and Convection problems in Fluid Mechanics. He has published more than seventy papers in referred international journals. He visited University of Glasgow, Scotland, UK under INSA bilateral exchange programme during 2009. Recently, he has visited Institute of Mechanics, Ruhr University, Bochum, Germany.

Received September 2010

Accepted February 2011

Final acceptance in revised form March 2011 